Artículo Original

\title{
Caracterización de la atención a distancia, preferencias y utilidad de redes sociales de los usuarios de Teletón Chile, en el período junio-agosto de 2020 , con motivo de la contingencia de COVID-19, estudio descriptivo transversal
}

ÓSCAR URREJOLA ${ }^{1}$, MAGALY DÍAZ ${ }^{1}$, NATALIA PÉREZ ${ }^{1}$, ZOIA PEDRERO ${ }^{2}$, VERÓNICA TORRES ${ }^{3}$, MACARENA RIVAS $^{4}$.

'Subdirección de Investigación, Teletón Chile.

2Instituto Teletón

Coquimbo

${ }^{3}$ Dirección Médica Nacional. ${ }^{4}$ Subdirección de Servicios Sociales y Comunitarios.

Correspondencia a: Macarena Rivas mrivas@teleton.cl

Recibido: 19 de abril de 2021 Aceptado: 18 de junio de

\begin{abstract}
Characterization of remote care, preferences and usefulness of social networks of Teletón Chile patients, in the period June-August 2020, on COVID-19 contingency, descriptive cross-sectional study
\end{abstract}

Introduction: Due to the COVID-19 pandemic and to establish continuity in the treatment of users and families, Teletón Chile implemented a survey to describe the demographic, health, social and environmental characteristics, associated factors, distance care and associated problems due to COVID-19. Objective: To describe stance Attention (DA) service implemented by Teleton Chile, and the preferences and usefulness of users' social networks (RRSS). Materials and Methods: A cross-sectional descriptive study of a census type which wasdeveloped through the application of a survey to characterize the DA and RRSS service, and includes active Teletón users as of April 30, 2020. Results: Results obtained between June and August 2020 are presented, representing $31.13 \%(\mathrm{n}=8,202)$ of the total population served; $74.54 \%$ of the survey participants indicated having received of which $82.02 \%$ achieved compliance with the prescribed indications, the main reason for non-compliance was not being contacted by the medical-therapeutic area for the DA modality $(36.55 \%)$. The main benefits of DA are: increased accessibility to care $(44.13 \%)$ and reduction in transportation problems $(17.67 \%)$. The main concern is the loss of the amount of face-to-face care (34.41\%). Conclusions: DA is considered useful for the continuity of rehabilitation programs. The use of social networks for contact and follow-up is also relevant. Finally, to implement this service, it is necessary to consider the personal needs of the users for a remote rehabilitation.

Key words: Distance care, telerehabilitation, disability, COVID-19, home rehabilitation. 


\section{RESUMEN}

Introducción: Producto de la pandemia por COVID-19 y para establecer continuidad a los tratamientos de usuarios y familias, Teletón Chile implementó una encuesta para describir las características demográficas, de salud, sociales, ambientales, factores asociados, atención a distancia y problemas asociados por contingencia COVID-19. Objetivo: Describir el servicio de atención a distancia (AD) implementado por Teletón Chile, preferencias y utilidad de redes sociales (RR.SS.) de los usuarios. Materiales y Métodos: Estudio descriptivo transversal de tipo censal a usuarios activos de Teletón al 30 de abril de 2020 desarrollado a través de la aplicación de una encuesta para caracterizar el servicio de AD y RR.SS. Resultados: Se presentan resultados obtenidos entre junio y agosto de 2020 , que representan al $31,13 \%(n=8.202)$ de la población total atendida; El 74,54\% de los participantes de la encuesta señalan haber recibido $\mathrm{AD}$, de los cuales un $82,02 \%$ logra el cumplimiento de las indicaciones siendo la principal razón de no cumplimiento no ser contactado para la modalidad AD desde el área médico-terapéutica (36,55\%). Los principales beneficios de la AD son: aumento de la accesibilidad en la atención (44,13\%) y disminución de las problemáticas del traslado $(17,67 \%)$. La preocupación principal es la pérdida de la cantidad de atenciones presenciales $(34,41 \%)$. Conclusiones: $\mathrm{La}$ $\mathrm{AD}$ es considerada de utilidad para la continuidad de los programas de rehabilitación. Asimismo, toma relevancia el uso de redes sociales para contacto y seguimiento. Finalmente, para implementar este servicio se requiere considerar las necesidades personales de los usuarios para una rehabilitación remota.

Palabras clave: Atención a distancia, telerehabilitación, discapacidad, COVID-19, rehabilitación en el hogar.

\section{Introducción}

La discapacidad es un término genérico que comprende deficiencias, limitaciones de actividad y restricciones para la participación, siendo el resultado negativo de la interacción entre personas con un problema de salud y sus factores personales y ambientales ${ }^{1}$. En Chile, la Ley 20.422, que establece normas sobre igualdad de oportunidades e inclusión social de personas con discapacidad, define a las personas con discapacidad como aquellas que teniendo una o más deficiencias físicas, mentales, sea por causa psíquica o intelectual, o sensoriales, de carácter temporal o permanente, al interactuar con diversas barreras presentes en el entorno, ven impedida o restringida su participación plena y efectiva en la sociedad, en igualdad de condiciones con las demás ${ }^{2}$.

Según el Informe Mundial de la Discapa- $\operatorname{cidad}^{3}$ se estima que más de mil millones de personas en el mundo viven con algún tipo de discapacidad, lo que equivale a un $15 \%$ de la población mundial al año 2010. En relación a la población infantil, el informe declara que los niños de las familias más pobres y pertenecientes a etnias minoritarias tienen un mayor riesgo de estar en una situación de discapacidad $^{3}$. A nivel nacional, la Encuesta Nacional de Discapacidad II (ENDISC II), declara que la población en situación de discapacidad en Chile alcanza el 16,7\% de la población general, elevándose este porcentaje a un $20 \%$ en la población adulta (18 años y más) y disminuyendo a un 5,8\% en la población infantil (2 a 17 años $)^{4}$.

Desde el 11 de marzo de 2020 que la enfermedad por coronavirus (COVID-19) ${ }^{5}$ fue declarada pandemia por la Organización Mundial de la Salud (OMS) ${ }^{6}$. A raíz de ello, 
existe preocupación por las condiciones de vulnerabilidad que puedan estar presentando las personas en situación de discapacidad. Es así, como han surgido investigaciones acerca de los posibles efectos secundarios de la pandemia por COVID-19 en personas con discapacidad $^{7}$, tales como problemas emocionales $^{8}$ y de aumento en brechas de salud durante el periodo de distanciamiento social y de disminución de atenciones de salud en formato presencial ${ }^{9}$.

Una estrategia para asegurar la accesibilidad de tratamientos de rehabilitación durante el periodo de distanciamiento social ha sido el uso de la telerehabilitación ${ }^{10}$. Esta es una rama de la telemedicina, a través de la cual se realizan intervenciones de rehabilitación utilizando tecnologías de la información y comunicación (TIC) ${ }^{11}$. Según la Organización Panamericana de Salud (OPS), esta herramienta permitiría a los usuarios beneficiarse de consultas remotas, garantizando una mayor protección a los grupos en situación de vulnerabilidad, junto con permitir la continuidad asistencial en el caso de los tratamientos de enfermedades crónicas o de largo plazo, donde la no interrupción de tratamiento es la clave para el éxito ${ }^{12}$. Sin embargo, la telerehabilitación puede verse limitada si no es posible el acceso a la tecnología, a material para realizar la rehabilitación, a capacitación en el uso de TIC tanto del equipo de salud como de los usuarios, entre otras situaciones ${ }^{12}$.

En el contexto local, los Institutos Teletón (IT) Chile, han impulsado la atención a distancia como principal mecanismo de continuidad de tratamiento durante el periodo de pandemia COVID-19, realizando contacto con sus usuarios a nivel nacional mediante el uso de llamadas telefónicas, videollamadas y telerehabilitación con la plataforma Teletón en Tu Casa ${ }^{13}$.

Dada la necesidad de obtener información sobre la atención a distancia (AD) que Teletón Chile ha implementado a raíz de la pandemia, para dar continuidad a los tratamientos de sus usuarios y sus familias a nivel nacional, es que la institución desarrolla durante el año 2020 una encuesta de caracterización que permitiera describir las características demográficas, de salud, sociales, ambientales, factores asociados, atención a distancia y problemas asociados por contingencia COVID-19 de los usuarios de los Institutos Teletón de Chile (Encuesta Teletón 2020). Dentro de los aspectos abordados por esta encuesta, se consultó acerca del servicio de atención a distancia (AD) implementado durante la pandemia y la preferencia y utilidad de las RR.SS. utilizadas por Teletón.

Considerando esta información, se plantea el objetivo de describir el servicio de atención a distancia que han recibido los usuarios activos de Teletón Chile, a nivel nacional, en el periodo junio-agosto del año 2020, con el propósito de dar a conocer una experiencia nacional de atención a distancia en el área de la neuro rehabilitación infanto-juvenil, e identificar las necesidades o brechas, referidas por los usuarios, que permitan mejorar la calidad de este servicio a mediano y largo plazo.

\section{Pacientes y Métodos}

Se realizó un estudio descriptivo de corte transversal de tipo censal para describir las características del servicio de atención a distancia, preferencias y utilidad de RR.SS., módulo que pertenece a uno de los 9 que constituían la Encuesta Teletón 2020, donde se invitó a participar a 26.590 usuarios activos al 30 de abril de 2020 , en un período de trabajo de campo entre junio y octubre de 2020. Se excluyeron de este estudio aquellos usuarios que no contaban con registro de número telefónico ni correo electrónico en el sistema de información clínica de Teletón. Se presentan sólo resultados del módulo de AD y RR.SS. de la Encuesta Teletón 2020 obtenidos entre junio y agosto de 2020 .

El estudio en su totalidad contó con la aprobación del Comité Ético Científico de la Sociedad Pro Ayuda al Niño Lisiado, Teletón $\mathrm{N}^{\circ} 112 / 2020$.

\section{Instrumento de recolección de datos}

Se analizaron los resultados relacionados con el servicio de atención a distancia implementado por Teletón y el impacto de la pandemia COVID-19 en la situación del usuario 
y su familia obtenidos del módulo de AD de la Encuesta Teletón 2020. Este módulo estaba conformado por 10 preguntas sobre AD y redes sociales (Tabla 1). La Encuesta Teletón
2020 contemplaba 9 módulos con un total de 151 preguntas. La Encuesta Teletón 2020 fue validada por criterio experto en 3 rondas de preguntas hasta llegar a un $100 \%$ de acuerdo

Tabla 1. Variables del estudio

\begin{tabular}{|c|c|c|}
\hline Ítems & Pregunta & Alternativas de respuesta \\
\hline $\begin{array}{l}\text { Atención a } \\
\text { distancia de } \\
\text { Teletón }\end{array}$ & $\begin{array}{l}\text { ¿Desde el inicio de la pandemia por } \\
\text { COVID-19 ha recibido atención a distancia } \\
\text { de Teletón? }\end{array}$ & $\begin{array}{l}\text { 1. Sí } \\
\text { 2. No } \\
\text { 3. No sabe }\end{array}$ \\
\hline $\begin{array}{l}\text { Utilidad de la } \\
\text { orientación o } \\
\text { atención entregada }\end{array}$ & $\begin{array}{l}\text { Si ha recibido contacto de alguno de los } \\
\text { profesionales o personal de Teletón, ¿la } \\
\text { orientación o atención entregada ha sido } \\
\text { de utilidad para usted? }\end{array}$ & $\begin{array}{l}\text { 1. Sí } \\
\text { 2. No } \\
\text { 3. No sabe }\end{array}$ \\
\hline $\begin{array}{l}\text { Cumplimiento de } \\
\text { las indicaciones } \\
\text { que le entregaron }\end{array}$ & $\begin{array}{l}\text { Si algún profesional del área terapéutica, } \\
\text { le ha entregado orientación para desa- } \\
\text { rrollar actividades en el hogar, ¿usted ha } \\
\text { podido cumplir con esas indicaciones? }\end{array}$ & $\begin{array}{l}\text { 1. Sí } \\
\text { 2. No }\end{array}$ \\
\hline $\begin{array}{l}\text { Respuesta No, } \\
\text { Razones de no } \\
\text { seguimiento de } \\
\text { indicaciones }\end{array}$ & $\begin{array}{l}\text { Si la respuesta anterior es NO, ¿cuáles } \\
\text { son las razones por las que no ha podido } \\
\text { seguir las indicaciones en el hogar? }\end{array}$ & $\begin{array}{l}\text { 1. No ha sido contactado o atendido por profesional área } \\
\text { terapéutica } \\
\text { 2. Contexto (espacio físico, falta de tiempo, materiales, } \\
\text { salud del cuidador) } \\
\text { 3. No ha recibido indicaciones de actividades en el hogar } \\
\text { 4. Problemas de salud o condición del paciente } \\
\text { 5. La información que entregaron es insuficiente } \\
\text { 6. No requiere } \\
\text { 7. Otro }\end{array}$ \\
\hline $\begin{array}{l}\text { Horario factible de } \\
\text { atención }\end{array}$ & $\begin{array}{l}\text { Considerando que la atención a distancia, } \\
\text { se proporciona mientras usted está en } \\
\text { su hogar, ¿en qué horarios le sería más } \\
\text { factible recibir la atención de algún profe- } \\
\text { sional de Teletón? }\end{array}$ & $\begin{array}{l}\text { 1. En la mañana } \\
\text { 2. A mediodía } \\
\text { 3. En la tarde } \\
\text { 4. Vespertino } \\
\text { 5. En cualquier horario, si se comunica previamente } \\
\text { 6. Prefiero que no me llamen }\end{array}$ \\
\hline $\begin{array}{l}\text { Es favorable contar } \\
\text { con programa } \\
\text { de atención } \\
\text { a distancia } \\
\text { complementaria }\end{array}$ & $\begin{array}{l}\text { Visualizando hacia el futuro, ¿usted con- } \\
\text { sidera que sería favorable para los pacien- } \\
\text { tes de Teletón contar con un programa de } \\
\text { atención a distancia que complemente la } \\
\text { atención que se realiza en los Institutos? }\end{array}$ & $\begin{array}{l}\text { 1. Sí } \\
\text { 2. No } \\
\text { 3. No sabe }\end{array}$ \\
\hline $\begin{array}{l}\text { Beneficio de plan a } \\
\text { distancia a la oferta } \\
\text { actual }\end{array}$ & $\begin{array}{l}\text { ¿Cuál cree usted sería el beneficio de } \\
\text { un plan a distancia a la oferta actual de } \\
\text { atención de Teletón?* }\end{array}$ & $\begin{array}{l}\text { 1. Aumenta la accesibilidad de atención } \\
\text { 2. Crea una alternativa para disminuir ausentismos y } \\
\text { tiempos de espera } \\
\text { 3. Entrega respuestas oportunas y resuelve dudas de } \\
\text { manera continua } \\
\text { 4. Potencia la participación y organización de los tiempos } \\
\text { de la familia } \\
\text { 5. Disminuye las problemáticas del traslado a los Institutos } \\
\text { 6. Permite proteger su salud en tiempos invernales y de } \\
\text { pandemia COVID-19 } \\
\text { 7. No sabe (no logra identificar beneficios) o no entiende } \\
\text { 8. Prefiere atención presencial }\end{array}$ \\
\hline
\end{tabular}


Tabla 1. Variables del estudio (continuación)

\begin{tabular}{|c|c|c|}
\hline Ítems & Pregunta & Alternativas de respuesta \\
\hline $\begin{array}{l}\text { Aspectos } \\
\text { preocupantes de la } \\
\text { modalidad }\end{array}$ & $\begin{array}{l}\text { Si Teletón implementara un plan de } \\
\text { atención a distancia que complemente } \\
\text { la atención presencial de los Institutos, } \\
\text { ¿qué aspectos le preocuparían en esta } \\
\text { modalidad de atención?* }\end{array}$ & $\begin{array}{l}\text { 1. Perder la calidad y calidez de la atención } \\
\text { 2. Perder las atenciones presenciales terapéuticas directas } \\
\text { 3. Tener problemas de conectividad, disponibilidad de } \\
\text { equipamiento tecnológico y uso de plataformas } \\
\text { 4. Contar con las habilidades para la comprensión e } \\
\text { implementación eficiente y efectiva del plan de re- } \\
\text { habilitación en el hogar (empoderamiento del rol del } \\
\text { cuidador) } \\
\text { 5. No tener condiciones en el hogar para recibir la } \\
\text { atención a distancia y/o para desarrollar la actividad } \\
\text { terapéutica de manera adecuada } \\
\text { 6. No aplica }\end{array}$ \\
\hline $\begin{array}{l}\text { Contenido de redes } \\
\text { sociales mantiene } \\
\text { informado al } \\
\text { paciente y/o } \\
\text { cuidador. }\end{array}$ & $\begin{array}{l}\text { ¿El contenido publicado en estas redes } \\
\text { sociales mantiene informado al paciente } \\
\text { y/o cuidador sobre el quehacer de } \\
\text { Teletón? }\end{array}$ & $\begin{array}{l}\text { 1. Siempre } \\
\text { 2. Casi siempre } \\
\text { 3. A veces } \\
\text { 4. Casi nunca } \\
\text { 5. Nunca }\end{array}$ \\
\hline $\begin{array}{l}\text { Preferencia de } \\
\text { recepción de } \\
\text { información sobre } \\
\text { quehacer Teletón }\end{array}$ & $\begin{array}{l}\text { ¿En cuál de las siguientes redes sociales } \\
\text { el paciente y/o cuidador preferiría recibir } \\
\text { información sobre el quehacer de } \\
\text { Teletón? }\end{array}$ & $\begin{array}{l}\text { 1. Facebook } \\
\text { 2. Instagram } \\
\text { 3. Twitter } \\
\text { 4. Linkedln } \\
\text { 5. Youtube } \\
\text { 6. Spotify } \\
\text { 7. TikTok } \\
\text { 8. Flickr } \\
\text { 9. En ninguna, no uso redes sociales }\end{array}$ \\
\hline
\end{tabular}

*Estas preguntas fueron respondidas a través de texto libre y fueron categorizadas para su análisis.

para ser luego piloteada en 30 usuarios de la población objetivo pertenecientes a distintos Institutos Teletón (IT), para llegar a la versión final de la encuesta. La encuesta se diseñó para ser respondida por los usuarios mayores de 18 años o por el representante legal de los usuarios menores de 18 años y de aquellos, mayores de 18 años que, debido a su condición, no podían responder la encuesta. Fueron excluidos los pacientes activos de los Institutos Teletón Chile que no contaban con registro de número telefónico ni correo electrónico de contacto en el Sistema de Información Clínica (SIC). Para la aplicación del instrumento a distancia se utilizó la plataforma LimeSurvey (versión 3.17.0). Los usuarios accedieron a la encuesta a través de un link enviado por correo electrónico y mensajería de texto. Los usuarios y representantes legales que aceptaron parti- cipar del estudio firmaron el consentimiento informado a través del mismo formulario. Debido al formato de aplicación en línea de la encuesta, las personas podían enviar la encuesta sin responder todas las preguntas por lo que podría haber variaciones en el número total de respuestas.

\section{Análisis estadístico}

Las variables de estudio analizadas se resumen en Tabla 1. La plataforma LimeSurvey permitió exportar los resultados directamente en una planilla Excel. El análisis de los datos se realizó mediante estadística descriptiva a través del software Stata v15.0, calculando medidas de resumen (promedio, desviaciones estándar, mínimos, máximos, entre otros), y construyendo tablas de frecuencias y gráficos para ilustrar los resultados de la investigación. 


\section{Resultados}

Del total de usuarios de Teletón Chile, activos al 30 de abril del año 2020, en el periodo junio-agosto del mismo año, 8.202 (31,13\%) aceptaron participar y respondieron la encuesta. De estas encuestas, el 5,82\% $(\mathrm{n}=477)$ fue respondida por usuarios, el $84,75 \%(\mathrm{n}=6.951)$ fue respondida por la madre, $5,97 \%(\mathrm{n}=490)$, por el padre, y el 3,46\% $(\mathrm{n}=284)$ restante, por el cuidador u otro representante legal de los usuarios.

En relación con las características sociodemográficas de los usuarios, se reportó una

Tabla 2. Caracterización sociodemográfica de la población de estudio

\begin{tabular}{|llcc|}
\hline Variable & Categoría & $\mathbf{n}$ & $\%$ \\
\hline \multirow{4}{*}{ Edad } & Mujer & 3.670 & 44,75 \\
& Hombre & 4.532 & 55,25 \\
& $\bar{x} \pm$ DE & $10,2 \pm 6,3$ & \\
& mínimo & 0,2 & \\
Instituto & máximo & 36,1 & \\
Teletón & Arica & 132 & 1,61 \\
& Iquique & 150 & 1,83 \\
& Antofagasta & 365 & 4,45 \\
& Calama & 262 & 3,19 \\
& Atacama & 266 & 3,24 \\
& Coquimbo & 551 & 6,72 \\
& Valparaíso & 866 & 10,56 \\
& Santiago & 2.847 & 34,71 \\
& Del Maule & 394 & 4,80 \\
& Concepción & 976 & 11,90 \\
& Temuco & 599 & 7,30 \\
& Valdivia & 157 & 1,91 \\
& Puerto Montt & 561 & 6,84 \\
& Aysén & 76 & 0,93 \\
& Total & 8.202 & 100 \\
\hline
\end{tabular}

media de edad de 10,2 $\pm 6,3$ años y el 55,25\% correspondió a usuarios de sexo masculino (Tabla 2). El porcentaje promedio de respuestas obtenidas a nivel nacional -en relación con el total de usuarios-, fue un $31,13 \%$ con un rango de respuesta por IT desde $12,36 \%$ a $44,86 \%$.

\section{Atención a distancia}

Con respecto a la cobertura del servicio de $\mathrm{AD}$, el 74,59\% $(\mathrm{n}=6.118)$ de los usuarios ha recibido el servicio en el período estudiado. De éstos, el 97,16\% ( $\mathrm{n}=5.944)$ consideró que fue de utilidad. Asimismo, el 82,02\% $(\mathrm{n}=5.018)$ dio cumplimiento a las indicaciones en el hogar entregadas por los profesionales del área terapéutica (Tabla 3). Sin embargo, el $15,07 \%(n=922)$ restante, que no pudo dar cumplimiento a las indicaciones, indicó como principales razones no haber sido contactado para la modalidad de $\mathrm{AD}$ por profesionales del área médico-terapéutica $(36,55 \%)$, no contar con espacio, tiempo, materiales o persona que se haga cargo de realizar la intervención $(24,08 \%)$, y no haber recibido indicaciones para realizar actividad terapéutica en el hogar (16,70\%) (Tabla 4).

En cuanto a los beneficios percibidos sobre la AD, destacaron el aumento de la accesibilidad para la atención $(44,13 \% ; n=3.085)$, la disminución de las problemáticas de traslado $(17,67 \% ; n=1.235)$ y las facilidades para la participación y organización de los tiempos familiares $(14,80 \% ; n=1.035)$ (Figura 1). Por otro lado, en cuanto a las principales preocupaciones respecto a esta modalidad de atención, destacaron el miedo a perder atenciones presenciales $(34,41 \% ; n=669)$ y el temor a perder la calidez y calidad de la atención brindada por Teletón $(20,32 \% ; n=395)$.

Tabla 3. Servicio de atención a distancia entregado por Teletón

\begin{tabular}{|c|c|c|c|c|c|c|c|c|c|}
\hline \multirow{2}{*}{$\begin{array}{l}\text { Ítems de preguntas } \\
\text { de módulo } A D\end{array}$} & \multicolumn{2}{|c|}{ Sí } & \multicolumn{2}{|c|}{ No } & \multicolumn{2}{|c|}{ No sabe } & \multicolumn{2}{|c|}{ No responde } & \multirow{2}{*}{$\begin{array}{c}\text { Total } \\
\mathbf{n}\end{array}$} \\
\hline & $\mathbf{n}$ & $\%$ & $\mathbf{n}$ & $\%$ & $\mathbf{n}$ & $\%$ & $\mathbf{n}$ & $\%$ & \\
\hline Ha recibido $A D$ & 6.118 & 74,59 & 2.021 & 24,64 & 42 & 0,51 & 21 & 0,26 & 8.202 \\
\hline Ha sido útil la $A D$ & 5.944 & 97,16 & 108 & 1,77 & 50 & 0,82 & 16 & 0,26 & 6.118 \\
\hline Cumplimiento de indicaciones & 5.018 & 82,02 & 922 & 15,07 & 0 & 0,00 & 178 & 2,91 & 6.118 \\
\hline AD como complemento de AP & 7.029 & 85,70 & 552 & 6,73 & 544 & 6,63 & 77 & 0,94 & 8.202 \\
\hline
\end{tabular}

Abreviaturas: AD: Atención a distancia; AP: Atención presencial. 
Finalmente, la gran mayoría de los participantes $(85,70 \% ; n=7.029)$, consideró favorable que Teletón cuente con un servicio de atención a distancia permanente (Tabla 3), con un horario de atención de preferencia durante la tarde $(51,94 \% ; n=4.260)$ (Tabla 5), con el fin de complementar la atención presencial del programa de rehabilitación.

\section{Redes sociales Teletón}

Teletón mediante sus redes sociales (RR.SS.), comparte información para los usuarios y/o cuidadores sobre el quehacer institucional, en este contexto, se consultó sobre la utilidad del contenido publicado y la preferencia de RR.SS. para recibirlo. De las 4.687 respuestas recibidas el $46,60 \%$

Tabla 4. Razones de no cumplimiento de indicaciones entregadas por profesionales del área terapéutica

\begin{tabular}{|lcc|}
\hline Razones de no cumplimiento & $\mathbf{n}$ & $\%$ \\
\hline No ha sido contactado por profesional del área médico-terapéuticas & 337 & 36,55 \\
\hline Contexto (espacio físico, falta de tiempo, materiales, salud del cuidador) & 222 & 24,08 \\
\hline No ha recibido indicaciones de actividades en el hogar & 154 & 16,70 \\
No se siente capacitado para hacerlo solo/a & 55 & 5,97 \\
\hline Problemas de salud o condición del paciente & 36 & 3,90 \\
\hline La información que entregaron es insuficiente & 35 & 3,80 \\
\hline No requiere & 35 & 3,80 \\
\hline Otro & 31 & 3,36 \\
No responde & 17 & 1,84 \\
Total & 922 & 100 \\
\hline
\end{tabular}

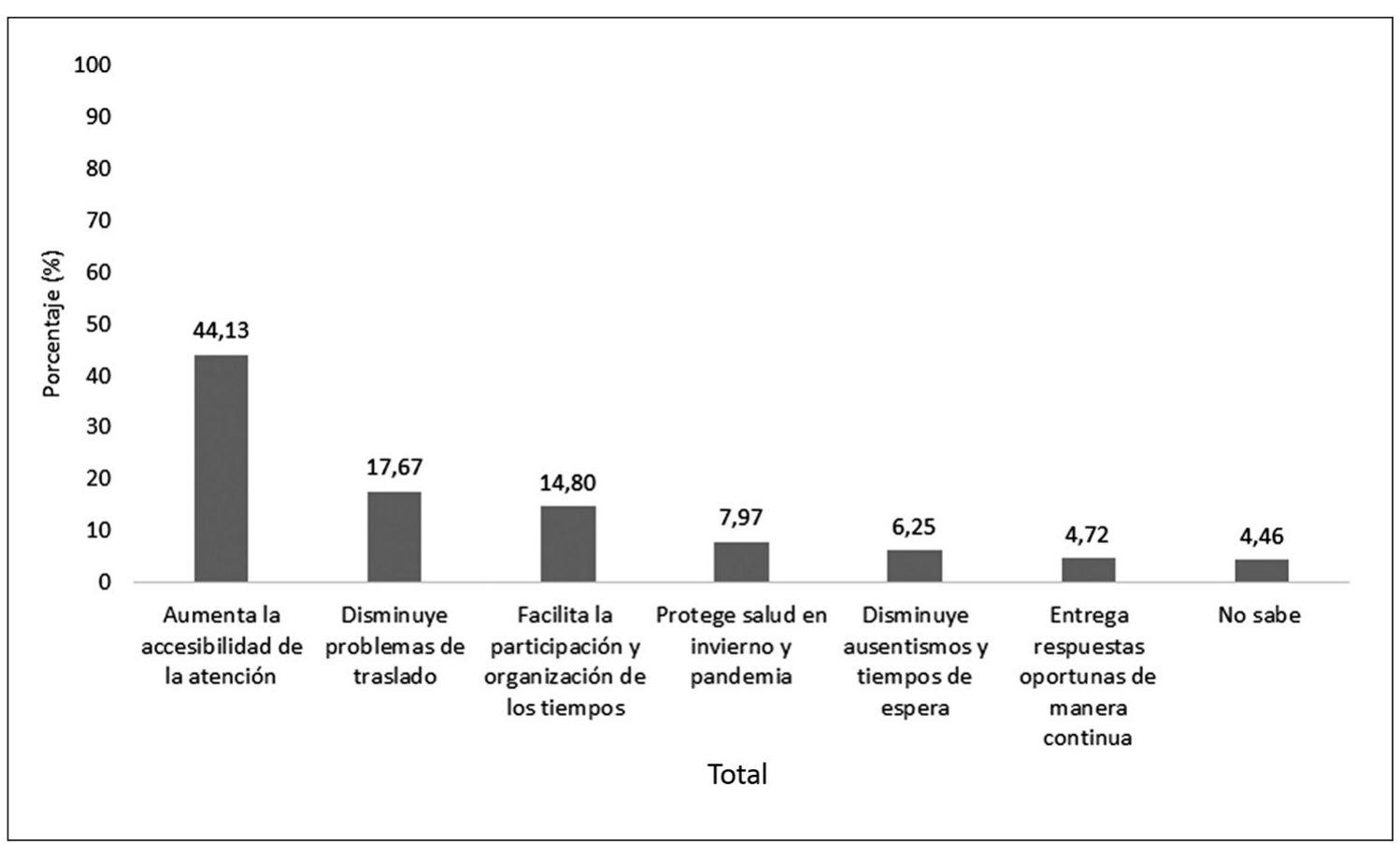

Figura 1. Percepción de beneficios de atención a distancia entregada por Teletón, a nivel nacional. 
Tabla 5. Horarios de preferencia para recibir atención a distancia

\begin{tabular}{|lrc|}
\hline Horarios de atención & $\mathbf{n}$ & $\%$ \\
\hline En la tarde & 4.260 & 51,94 \\
\hline En cualquier horario, si se comunica & 1.588 & 19,36 \\
\hline En la mañana & 1.135 & 13,84 \\
\hline A medio día & 900 & 10,97 \\
Vespertino & 203 & 2,48 \\
\hline Prefiero que no me llamen & 49 & 0,60 \\
No responde & 67 & 0,82 \\
Total & 8.202 & 100 \\
\hline
\end{tabular}

$(\mathrm{n}=2.184)$ de los encuestados consideró que el contenido publicado siempre es útil, en contraste a un 2,24\% $(n=105)$ que refiere no ser nunca de utilidad. Respecto a la RR.SS. de su preferencia, de las 4.682 respuestas recibidas el $65,08 \%(n=3.047)$ de los usuarios opta por Facebook, seguido de Instagram 27,34\% $(\mathrm{n}=1.280)$. Cabe destacar que existe un $1,92 \%(\mathrm{n}=90)$ de los encuestados que no utiliza ninguna RR.SS. de Teletón (Tabla 6).

Tabla 6. Utilidad y preferencia de las redes sociales de los usuarios para recibir información de Teletón

\begin{tabular}{|c|c|c|c|}
\hline Redes sociales, utilidad y preferencia & Categoría & $\mathbf{n}$ & $\%$ \\
\hline \multirow[t]{6}{*}{ Utilidad de publicación en RR.SS. } & Siempre & 2.184 & 46,60 \\
\hline & Casi siempre & 1.172 & 25,01 \\
\hline & A veces & 1.054 & 22,49 \\
\hline & Casi nunca & 172 & 3,67 \\
\hline & Nunca & 105 & 2,24 \\
\hline & Total & 4.687 & 100 \\
\hline \multirow[t]{8}{*}{ RR.SS. de preferencia para recibir información } & Facebook & 3.047 & 65,08 \\
\hline & Instagram & 1.280 & 27,34 \\
\hline & Youtube & 158 & 3,37 \\
\hline & Twitter & 70 & 1,50 \\
\hline & TikTok & 26 & 0,56 \\
\hline & Linkedln & 11 & 0,23 \\
\hline & En ninguno, no uso redes sociales & 90 & 1,92 \\
\hline & Total & 4.682 & 100 \\
\hline
\end{tabular}

Abreviaturas: RR.SS.: Redes sociales.

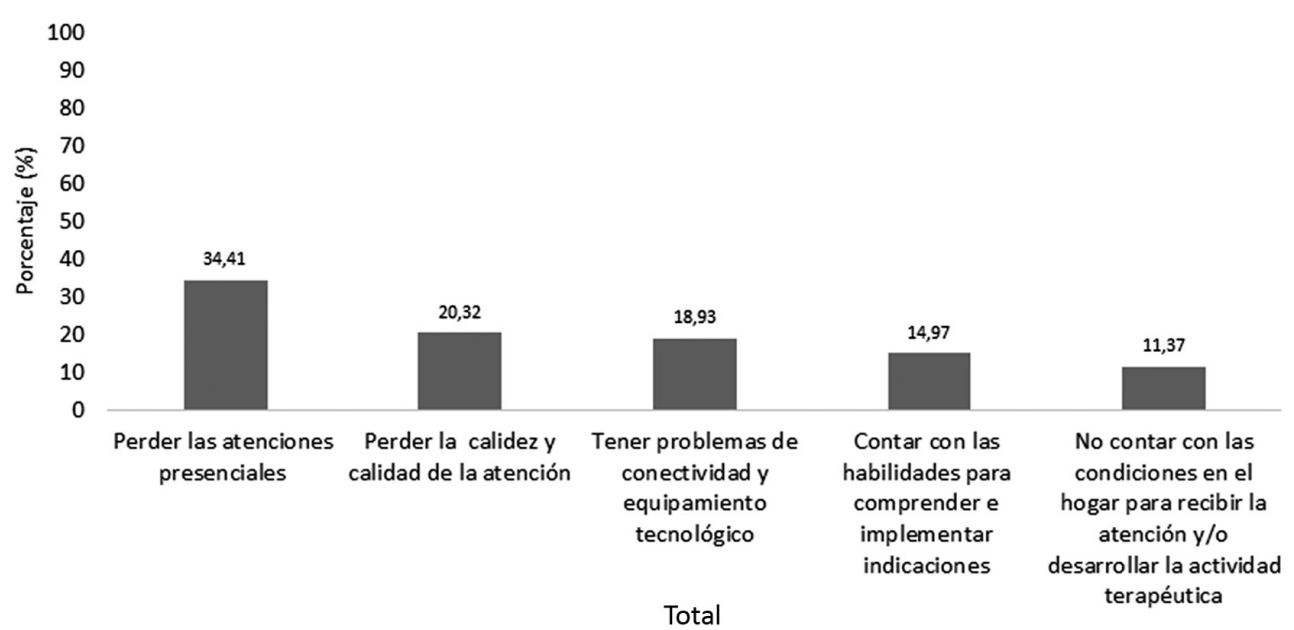

Figura 2. Preocupaciones sobre la atención a distancia entregada por Teletón, a nivel nacional. 


\section{Discusión}

Los resultados preliminares de la encuesta representan el 31,13\% ( $n=8.202)$ de los usuarios activos de Teletón Chile. Por el nivel de participación es la encuesta con mayor número de respuestas de personas en situación de discapacidad en la población infanto juvenil en Chile ${ }^{4,14}$.

El uso de la atención de salud a distancia se ha masificado en el periodo de COVID-19, emergiendo como una herramienta vital para proveer la continuidad de terapias en niños con discapacidad a través del curso de la pandemia ${ }^{15}$. La aplicación de la Encuesta Teletón 2020 y el análisis de los datos obtenidos del módulo de AD, para el período junio-agosto del año 2020, demostraron que el servicio de AD presentó una cobertura del $74,59 \%$, considerándose de utilidad por los usuarios y sus familias. Se logró un $82,02 \%$ de cumplimiento de las indicaciones entregadas por el área médicoterapéutica, siendo las principales razones para no cumplirlas, no tener espacio, tiempo y/o materiales en el hogar para realizarlas y no haber recibido indicaciones para realizar la actividad terapéutica en el hogar. La AD es considerada beneficiosa para aumentar el acceso a la atención lo que es coincidente con el estudio de Nulle realizado el año 2020 que la señala como una herramienta vital para proveer la continuidad de terapias en niños con discapacidad. Además, la AD es beneficiosa al disminuir las problemáticas económicas y de tiempo durante los traslados hacia los institutos como lo indica Nulle ${ }^{15}$. No obstante, lo anterior, existen preocupaciones de los usuarios, en término de mantener la calidad y calidez del servicio junto con proteger el número de atenciones presenciales tal como lo reporta Suffren ${ }^{16}$ y la OMS enfatiza el mantener la calidad de la atención ${ }^{17}$.

En relación con la cobertura de atención de usuarios en Teletón, lo reportado en la literatura es de un $49,5 \%{ }^{18}$, sin embargo, en nuestro estudio la cobertura del servicio de atención a distancia alcanzó un $74,59 \%$. Por otro lado, un $15,07 \%$ de los usuarios y/o cuidadores reportaron que no han logrado dar cumplimiento a las indicaciones para el hogar y de estos un 5,97\% refiere que el motivo es por la falta de capacitación de los cuidadores, lo que es similar a lo reportado por Proven$\mathrm{zi}^{19}$, que esta falta de capacitación se puede explicar por lagunas de alfabetización web y dificultades para seguir las instrucciones dadas por el terapeuta.

Por otro lado, los resultados dan cuenta que el $65,08 \%$ prefieren recibir información de Teletón a través de Facebook Teletón, lo que podría constituirse en un recurso de apoyo en la implementación permanente de un servicio de atención a distancia. En el estudio de Hohenschurz-Schmidt ${ }^{20}$ se señala que se han creado grupos de apoyo profesional a través de esta red, lo que podría sugerir elementos de encuentro entre familias y profesionales para facilitar la interacción durante la entrega del servicio de atención a distancia. Por otro lado, el mismo estudio, recomienda el entrenamiento previo de los profesionales de salud en el uso de redes sociales con fines de atención a distancia.

Por otra parte, una de las limitaciones de este estudio fue el no incluir preguntas relacionadas a las competencias de los usuarios y sus familias respecto al uso de TIC para iniciar y optimizar los programas de AD para dar continuidad a su plan de rehabilitación.

Dentro de las proyecciones de este estudio, se han observado experiencias en que el uso de programas de rehabilitación basados en la $\mathrm{AD}$, han demostrado la efectividad en la función motora en niños con parálisis cerebral (PC), por lo que se sugiere la medición de la efectividad del servicio de AD en Teletón. Además, producto de los resultados proponemos explorar el uso de redes sociales para la rehabilitación, debido a que esta herramienta podría facilitar la interacción durante la entrega del servicio de $\mathrm{AD}^{20}$.

Finalmente, el aporte que entrega la investigación en la descripción del servicio de $\mathrm{AD}$ entregado en una situación de pandemia a los usuarios, podría ser relevante en términos de planificación sanitaria, pues el aislamiento físico como medida de control del aumento de la circulación viral, produce deterioro en el proceso de atención de las personas con limitaciones de movimiento er $s e^{1,10,12,20,21}$. 


\section{Conclusiones}

Los resultados obtenidos entre junio y agosto del módulo de AD de la Encuesta Teletón 2020 dan cuenta que la atención a distancia es considerada útil por los usuarios de Teletón y sus familias y puede ser beneficiosa para aumentar el acceso y continuidad de la atención y disminuir las dificultades del traslado de los pacientes a los centros de atención. En la AD toma relevancia el uso de redes sociales para el contacto y seguimiento de los usuarios y sus familias asimismo para la entrega de información y contenidos educativos. Se requiere de estudios futuros que permitan medir el efecto de este tipo de intervención en la funcionalidad y calidad de vida de los usuarios. Por otra parte, para el cumplimiento de las indicaciones terapéuticas en el hogar, en poblaciones más vulnerables, se requiere considerar las necesidades contextuales planteadas por los usuarios, en términos de capacitación, acompañamiento, espacio físico y equipamiento terapéutico.

\section{Agradecimientos}

Al equipo de colaboradores de Teletón y voluntariado que permitieron contactar a los usuarios y familias participantes del estudio, junto con el equipo de la Subgerencia de Tecnologías de la Información y Comunicaciones de Teletón Chile que permitieron el contacto on-line y dar soporte a la plataforma utilizada para recabar la información. Al equipo de Fundación Teletón que permitió la difusión de la encuesta a nivel nacional y, finalmente, agradecer a la Dirección Médica Nacional de Teletón Chile y vicepresidencia por el apoyo otorgado en la realización de la Encuesta Teletón 2020 y este estudio.

\section{Conflictos de interés}

Los investigadores declaran que no presentan conflictos de interés en relación con este estudio.

\section{Referencias}

1. Organización Mundial de la Salud [Internet] Clasificación Internacional del Funcionamiento, la Discapacidad y la Salud (CIF). 2001. Disponible en: https://apps.who. int/iris/bitstream/handle/10665/43360/9241545445_spa. pdf;jsessionid=5246009AD12D4390501B9E5274E1C6 D5? sequence $=1$.

2. Ley 20.422. [Internet] Establece normas sobre igualdad de oportunidades e inclusión social de personas con discapacidad. Santiago, Chile. 2010. Disponible en: https://www.bcn.cl/leychile/navegar?idLey=20422.

3. Organización Mundial de la Salud [Internet]. Reporte mundial de la discapacidad. 2011. Disponible en: https://www.who.int/teams/noncommunicablediseases/disability-and-rehabilitation/world-report-ondisability.

4. Instituto Nacional de Estadística [Internet] Estudio Nacional de la Discapacidad. Santiago, Chile: 2015. Disponible en: https://www.ine.cl/docs/default-source/ discapacidad/publicaciones-y-anuarios/primer-estudionacional-de-la-discapacidad-en-chile-endisc-cif-chile-2004/presentacion-resultados-estudio-nacional-dela-discapacidad.pdf?sfvrsn=3bcf2aec_2.

5. Lu R, Zhao X, Li J, Niu P, Yang B, Wu H, et al. Genomic characterisation and epidemiology of 2019 novel coronavirus: implications for virus origins and receptor binding. Lancet. 2020; 395 (10224): 565-74.

6. Cucinotta D, Vanelli M. WHO Declares COVID-19 a Pandemic. Acta Biomed. 2020; 91 (1): 157-60.

7. Papa SM, Brundin P, Fung VS, et al. Impact of the COVID-19 pandemic on Parkinson's disease and movement disorders. Movement Disorders. 2020.

8. Lima CKT, Carvalho PMM, Lima IAAS, Nunes JVAO, Saraiva JS, de Souza RI, et al. The emotional impact of Coronavirus 2019-nCoV (new Coronavirus disease). Psychiatry Res. 2020; 287: 112915.

9. Chávez MCM. Coronavirus y discapacidad: Una población muy vulnerable. Acta odontológica venezolana. 2020; 58 (1): 9-10.

10. Nuara A, Fabbri-Destro M, Scalona E, Lenzi SE, Rizzolatti G, Avanzini P. Telerehabilitation in response to constrained physical distance: an opportunity to rethink neurorehabilitative routines. J Neurol. 2021; 1-12.

11. Peretti A, Amenta F, Tayebati SK, Nittari G, Mahdi SS. Telerehabilitation: Review of the State-of-the-Art and Areas of Application. JMIR Rehabil Assist Technol. 2017; 4 (2).

12. Organización Panamericana de Salud [Internet] COVID-19: Salud digital facilitando la telerehabilitación 
2020. Disponible en: https://www.paho.org/es/documentos/covid-19-salud-digital-facilitando-telerehabilitacion.

13. Cubillos R. Informe de Jefe de Unidad de Tecnologías Asistivas, Teletón 2020.

14. Servicio Nacional de Discapacidad [Internet]. Estudio Nacional de la Discapacidad 2015. Disponible en: http:// endisc.senadis.cl/resultados.html.

15. Nulle J, Nelson VS. Video visits and access to care in pediatric rehabilitation therapies in the time of a pandemic. J Pediatr Rehabil Med 2020; 13 (3): 385-8.

16. Suffren S, Dubois-Comtois K, Lemelin J-P, St-Laurent $\mathrm{D}$, Milot T. Relations between child and parent fears and changes in family functioning related to COVID-19. Int. J. Environ. Res. Public Health 2021; 18: 1786. https// doi.org/10.3390/ijerph18041786.

17. Houtrow A, Harris D, Molinero A, Levin-Decanini T, Robichaud C. Children with disabilities in the United States and the COVID-19 pandemic. J Pediatr Rehabil Med. 2020; 13 (3): 415-24. doi: 10.3233/PRM-200769. PMID: 33185616.

18. Bova SM, Basso M, Bianchi MF, Savaré L, Ferrara G,
Mura E, et al. Impact of COVID-19 lockdown in children with neurological disorders in Italy. Disabil Health J. 2020:101053.

19. Provenzi L, Grumi S, Gardani A, Aramini V, Dargenio $\mathrm{E}$, Naboni $\mathrm{C}$, et al. Engaging with Families through On-line Rehabilitation for Children during the Emergency (EnFORCE) Group. Italian parents welcomed a telehealth family-centred rehabilitation programme for children with disability during COVID-19 lockdown. Acta Paediatr. 2021 Jan;110(1):194-196. doi: 10.1111/ apa.15636. Epub 2020 Nov 6. PMID: 33098116.

20. Hohenschurz-Schmidt D, Scott W, Park C, Christopoulos G, Vogel S, Draper-Rodi J. Remote management of musculoskeletal pain: a pragmatic approach to the implementation of video and phone consultations in musculoskeletal practice. Pain Rep. 2020; 5 (6): e878.

21. Longo E, de Campos AC, Schiariti V. COVID-19 Pandemic: Is This a Good Time for Implementation of Home Programs for Children's Rehabilitation in Low- and Middle-Income Countries? Phys Occup Ther Pediatr. 2020; 40 (4): 361-4. 